\title{
Mathematical model of the thermodynamic system during winter concreting of bored piles
}

\author{
Aleksey Mestnikov, Gavril Turantaev and Valeriy Fedorov ${ }^{*}$ \\ North-Eastern Federal University named M. Ammosov, Institute of Engineering \& Technology, \\ 677027 Belinsky Street 58, Russia
}

\begin{abstract}
The article discusses the development of a mathematical model of the thermodynamic system during winter concreting of bored piles. The processes of heat transfer based on the differential heat equation and the Stefan problem are described. A method for calculating the rationale for the parameters of the energy-saving and gentle thermal treatment of concrete using non-traditional sources of thermal energy is proposed. The solutions for using two-phase thermosyphons as heating elements are considered.
\end{abstract}

\section{Introduction}

Construction of buildings and structures in the regions of the Far North is carried out in harsh climatic and difficult traffic conditions [1]. Significant difficulties in construction also arise due to the dispersal of objects and the remoteness of materials and products from the centralized supply bases, the complexity of the rapid maneuvering of construction equipment and the limited energy resources [2]. In the construction of foundations, frameworks of buildings, columns, power lines, etc. Approximately 50\% of this volume falls on the winter season, when the outdoor temperature reaches $-50^{\circ} \mathrm{C}$ [3].

The widespread introduction in the construction of metal inventory formwork, the economic efficiency of which is ensured largely due to the high rate of its turnover, the increase in the specific proportion of thin-walled densely reinforced structures in the total volume of monolithic concrete and reinforced concrete requires a qualitatively new approach to the production technology of monolithic work in winter conditions, taking into account the specifics northern region $[4,5]$. Existing methods of winter concreting are often technologically difficult and do not always provide the necessary pace of construction [68].

With an increase in the volume of use of unified shield formwork forms, one of the most technologically advanced and effective method of winter concreting is a method of contact heating of concrete structures. The implementation of this method is achieved by heating concrete and reinforced concrete in thermoactive formwork with a fundamentally new heat distribution system based on heat pipes operating from any source of thermal energy $[3,6,9]$.

\footnotetext{
* Corresponding author: valeriyif.ykt@gmail.com
} 
Analysis of the existing methods of winter concreting revealed the effectiveness of the method of electrical heating of monolithic structures using various variants of thermoactive formwork forms [10-12]. As a heating element it is proposed to use a kind of heat pipe - a two-phase thermosyphon [3]. A two-phase thermosyphon is an evaporative-condensation device for heat transfer, in which latent heat of vaporization is transferred, due to evaporation of liquid in the heat supply zone and its vapor condensation in the retraction zone, and the closed circulation of the coolant is supported by gravitational forces $[3,13$ 17].

Based on the above, the purpose of the research is to develop a method for calculating the rationale for the parameters of the energy-saving and gentle thermal treatment of concrete using a two-phase thermosyphon.

\section{Methods}

In mathematical modeling, the classical equations of heat physics are used, which do not require proof of reliability $[18,19]$ : the differential equation of heat conduction; Stephen's condition; classical boundary conditions I (given the concrete temperature at the boundary), II (the intensity of heat flow at the boundary), III (heat transfer at the boundary according to Newton's law), IV (condition of thermal contiguity of two solids) of the genus. The processing of calculated and experimental data is performed on the programs MathCAD $2001 i$ and Microsoft office EXCEL.

\section{Results and Discussion}

A single bored pile (Fig. 1) is concreted with heat treatment of concrete using a two-phase thermosyphon, the annular evaporation chamber of which is located in the annular lower part of the lattice heater. The number of vertical elements $N$, the central angle between adjacent vertical elements $F=2 \pi / N$.

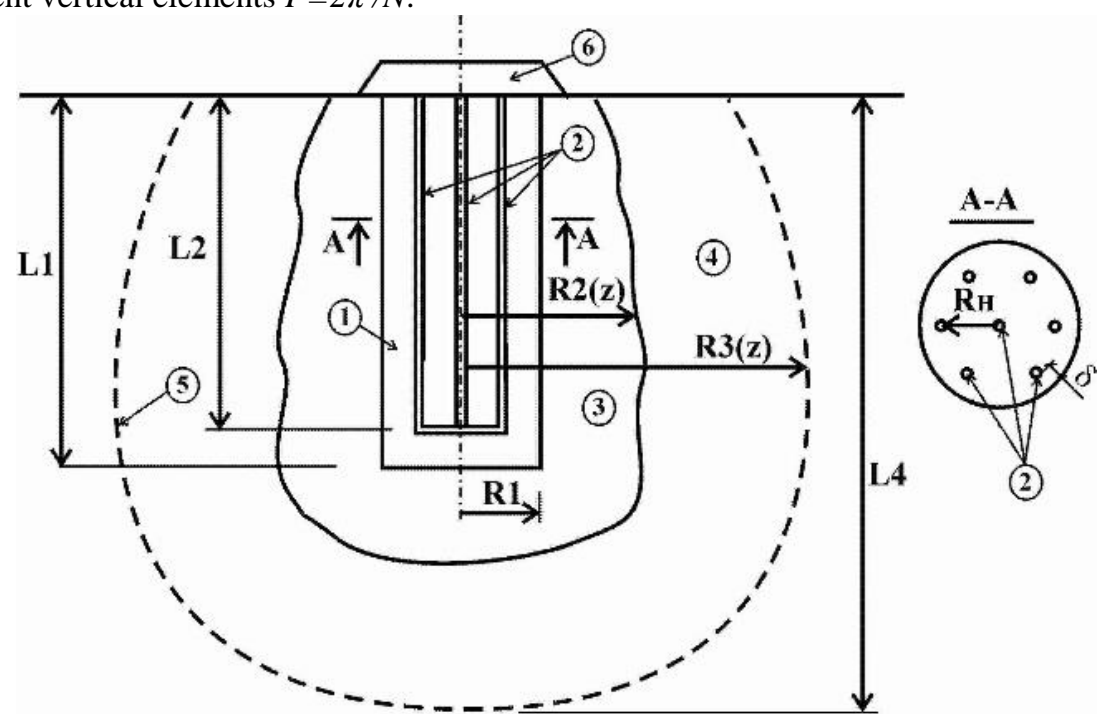

Fig. 1. Diagram of a single concreted bored pile: 1 - bored pile;

2 - vertical elements of a two-phase thermosyphon; 3 - thawed permafrost;

4 - permafrost soil mass around the pile; 5 - the boundaries of thermal influence of the pile;

6 - surface insulation 
The diameters of the heating elements: external $D_{H}$ and internal $D_{6}$. The distance from the center to the central peripheral vertical element of the two-phase thermosyphon $R_{n}$. The weather heat generation of vertical heat heaters, W/m, depends on the mode of conversion of thermal energy into the evaporation chamber of a two-phase thermosyphon and in the general case can be: a) continuous, not adjustable; b) continuous adjustable; c) discontinuous. The surface of the bored pile is insulated with a layer of insulation $\delta_{y}$ with thermal conductivity coefficients $\lambda_{y}$. Pile radius $R_{l}$, radius of thawing of permafrost soil $R_{2}(z)$, radius of thermal influence of the pile $R_{3}(z)$, height of the pile, depth of thermal influence of the pile $L_{3}$. With a sufficient margin of safety, the value of $L_{3}$, and in the future it will be refined by calculations. The movement of the $R_{2}(z)$ boundary is taken into account: in unbound soils using the classical Stefan condition $[3,18]$ :

$-\lambda_{1} \frac{\partial t_{1}\left(r=R_{2}, z, \tau\right)}{\partial r}+\lambda_{2} \frac{\partial t_{1}\left(r=R_{2}, z, \tau\right)}{\partial r}=\varepsilon \omega \rho_{2} \frac{d R_{2}(z)}{d \tau}$

where, index 1 refers to thawed ground, index 2 to frozen ground; $\varepsilon$ is the specific heat of the water-ice phase transitions; $\omega$ and $\rho_{2}$-respectively, the humidity in fractions of a unit and the density of the soil. In related using the differential heat equation with a volumedistributed phase-type heat source $[4,9,18]$ :

$\frac{\partial t(z, \varphi, r, \tau)}{\partial \tau}=\frac{\lambda_{1,2}}{C_{1,2}}\left(\begin{array}{c}\frac{\partial^{2} t(z, \varphi, r, \tau)}{\partial r^{2}}+\frac{1}{r} \frac{\partial t(z, \varphi, r, \tau)}{\partial r}+ \\ +\frac{\partial^{2} t(z, \varphi, r, \tau)}{\partial z^{2}}+\frac{1}{r^{2}} \frac{\partial^{2} t(z, \varphi, r, \tau)}{\partial \varphi^{2}}\end{array}\right) \pm \frac{W_{\phi}(\tau)}{C_{1,2}}$

where, $W_{\phi}(\tau)$ - volume-distributed phase heat source.

In contrast to the existing solution of the problem for a single bored pile [7, 9], the problem must be solved in a three-dimensional formulation, which uses a heater that is precise with coordinate reference $z \in L_{2} \wedge \tau \geq 0 \wedge \varphi=\varphi_{s}, s=1,2, \ldots, N$. The mathematical model of the temperature field in concrete for the problem in question is:

$\left\{\begin{array}{l}\frac{\partial t(x, \varphi, r, \tau)}{\partial \tau}=\frac{\lambda_{\sigma}}{C_{\sigma}}\left[\frac{\partial^{2} t}{\partial r^{2}}+\frac{1}{r} \frac{\partial t}{\partial r}+\frac{\partial^{2} t}{\partial z^{2}}+\frac{1}{r^{2}} \frac{\partial^{2} t}{\partial \varphi^{2}}\right]+\frac{W(\tau)}{C_{6}}+\frac{Q_{m c}(x, \varphi, r, \tau)}{C_{6}} \\ z \in L_{1} \wedge r \in R_{1} ; Q_{m c}>0 \wedge z \in L_{2} \wedge \varphi=\varphi_{s}, s=1,2, \ldots, N\end{array}\right.$

(3) - differential equation of heat conduction for a bored pile, taking into account the body-distributed heat source of exothermic type (in cylindrical coordinates) and thermal effect of the vertical heating element of a two-phase thermosyphon;

$\left\{\begin{array}{l}\frac{\partial t(x, \varphi, r, \tau)}{\partial \tau}=\frac{\lambda_{1,2}}{C_{1,2}}\left[\frac{\partial^{2} t}{\partial r^{2}}+\frac{1}{r} \frac{\partial t}{\partial r}+\frac{\partial^{2} t}{\partial z^{2}}+\frac{1}{r^{2}} \frac{\partial^{2} t}{\partial \varphi^{2}}\right] \pm \frac{W_{\phi}(\tau)}{C_{1,2}} \\ z \in L_{1} \wedge r \in\left(R_{1}<r<R_{3}\right) ;\left(L_{1}<z<L_{3}\right) \wedge r \in R_{3}\end{array}\right.$

(4) - differential equation of heat conduction for thawed and frozen soils, taking into account the volume-distributed source (drain) of phase type heat in the freezing (thawing) layer;

$\frac{\partial t(z, \varphi=0, r, \tau)}{\partial r}=0$ 
(5) - thermal symmetry condition;

$\frac{\partial t(z, \varphi, r=0, \tau)}{\partial r}=0$

(6) - condition of thermal symmetry on the geometric axis of the pile;

$\frac{\partial t\left(z, \varphi, r=R_{3}, \tau\right)}{\partial r}=0$

(7) - boundary condition at the junction of thermal influence of the pile;

$\left\{\begin{array}{l}\lambda_{\sigma} \frac{\partial t_{\sigma}\left(z, \varphi, r=R_{1}, \tau=0\right)}{\partial r}-\lambda_{2} \frac{\partial t_{2}\left(z, \varphi, r=R_{1}, \tau=0\right)}{\partial r}=\varepsilon \omega \rho_{2} \frac{d R_{2}(z)}{d \tau} \\ z \in L_{1} \wedge r=R_{1} \wedge \tau=0\end{array}\right.$

(8) - the Stefan condition for disconnected soils at the phase boundary at the first step of integration over time with approximation by an explicit difference scheme;

$\left\{\begin{array}{l}-\lambda_{1} \frac{\partial t_{1}\left(z, \varphi, r=R_{2}, \tau\right)}{\partial r}+\lambda_{2} \frac{\partial t_{2}\left(z, \varphi, r=R_{2}, \tau\right)}{\partial r}=\varepsilon \omega \rho_{2} \frac{d R_{2}(z)}{d \tau} \\ z \in L_{4}(\tau) \wedge r=R_{2} \wedge \tau>0\end{array}\right.$

(9) - Stefan condition for non-cohesive soils in the following (after the first) time integration steps;

$\left\{\begin{array}{l}-\lambda_{\sigma} \frac{\partial t_{\sigma}\left(z, \varphi, r=R_{\mathrm{H}}, \tau\right)}{\partial r}+\lambda_{\sigma} \frac{\partial t_{6}\left(z, \varphi, r=R_{\mathrm{H}}, \tau\right)}{\partial r}=Q_{m c} \\ z \in L_{2} \wedge \tau \geq 0 \wedge \varphi=\varphi_{s}, s=1,2, \ldots, N z \in l_{2}, \tau \geq 0\end{array}\right.$

(10) - boundary condition at the location of the vertical element of a two-phase thermosyphon; note that the left-hand side of (10) is not a zero algebraic sum, since the derivatives $\frac{\partial t_{\mathrm{B}}}{\partial r}$ when approaching $r=R_{\mathrm{H}}$ and away from $r=R_{\mathrm{H}}$ are not equal to each other;

$\left\{\begin{array}{l}-\lambda_{\sigma} \frac{\partial t_{\sigma}\left(z, \varphi, r=R_{\mathrm{H}}, \tau\right)}{\partial r}+\lambda_{1} \frac{\partial t_{1}\left(z, \varphi, r=R_{1}, \tau\right)}{\partial r}=0 \\ z \in L_{1} \wedge \tau>0 \wedge r=R_{1}\end{array}\right.$

(11) - boundary condition at the junction of the pile-soil;

$-\lambda_{\sigma} \frac{\partial t_{б}(z=0, \varphi, r, \tau)}{\partial z}=K_{\text {пр }}\left[t_{\sigma}(z=0, \varphi, r, \tau)-\theta_{\ni}\right], r \in R_{1}$

(12) - boundary condition from above for the pile (taking into account the thermal insulation of the pile surface); 
$-\lambda_{1,2} \frac{\partial t_{1,2}(z=0, \varphi, r, \tau)}{\partial z}=\alpha\left[t_{1,2}(z=0, \varphi, r, \tau)-\theta_{\ni}\right], R_{1}<r \leq R_{2}$

(13) - boundary condition from above for a snow-drenched soil massif surrounding a bored pile;

$t_{2}\left(z=L_{3}, \varphi, r, \tau\right)=$ cost $=t$

(14) - boundary condition below;

$\left\{\begin{array}{l}t_{6}(z, \varphi, r, \tau=0)=t_{\mathrm{H}}, z \in L_{1} \wedge r \in R_{1} \\ t_{1,2}(z, \varphi, r, \tau=0)=\varphi(z), z \in L_{1} \wedge R_{1}<r \leq R_{3} \\ \left(L_{1}<z \leq L_{3}\right) \wedge r \in R_{3}\end{array}\right.$

(15) - initial conditions;

$\left\{\begin{array}{l}q_{m c}=q_{m c}-\Delta q_{m c}, t_{\sigma}(z, \varphi, r, \tau) \geq t_{\max } \vee \sqrt{b^{2}-4 a c} \\ \frac{\partial t_{\sigma}(z, \varphi, r, \tau)}{\partial \tau} \geq\left(\frac{\partial t}{\partial \tau}\right)_{\max }\end{array}\right.$

(16) - maximum temperature control unit;

$q_{m c}=q_{m c}-\Delta q_{m c}, t_{\sigma}(z, \varphi, r, \tau) \leq t_{\text {min }}$

(17) - minimum temperature control unit;

$\left\{\begin{array}{l}q_{m c}=W ; p=2 k+1, k=1,2, \ldots, T \\ q_{m c}=0 ; p=2 k, k=1,2, \ldots, T\end{array}\right.$

(18) - control units for intermittent heat treatment of concrete;

$$
\left\{\begin{array}{l}
-\lambda_{\text {б }} \frac{\partial t_{\text {б }}\left(z, \varphi, r=R_{1}, \tau\right)}{\partial r}+\lambda_{1,2} \frac{\partial t_{1,2}\left(z, \varphi, r=R_{1}, \tau\right)}{\partial r}=q_{\text {то }}, z \in L_{1} \\
Q=\frac{q_{\text {пог }} \Delta z}{\left(R_{4} \Delta \varphi \Delta r \Delta z\right)} \\
Q=q_{\text {пог }} \sum \frac{l}{\left(2 \pi R_{4} L_{2} \Delta r\right)}
\end{array}\right.
$$

(19) - condition of thermal contact of disposable thermoactive formwork with bored pile concrete and soil.

Differential equations (1) and (2) are approximated by the implicit difference scheme of fractional steps of N. Yanenko [20]. According to this scheme, the time step is divided into three runs along spatial coordinates. At each sweep in the forward direction, the sweep coefficients are calculated, and in the reverse, the temperature at each coordinate node of the spatial grid. At the same time, to simplify the algorithm, equation (1) with the control of thermophysical constants, as well as the acceptance of the pile limits, is taken as the basis. For convenience of calculating the temperature field, the direction of the $\mathrm{z}$ axis is taken from top to bottom, which coincides with the geometric axis of the pile, the axis is counterclockwise, and the radius $r$ is with a deviation on the geometric axis of the pile. 
The numerical approximation of differential equations is performed using a special algorithm on certified software. The results of the calculations are given in $[3,6]$.

\section{Conclusions}

1. A method for heating monolithic concrete when concreting bored piles with a heating device of two-phase siphons working from any source of thermal energy is proposed.

2. Physical and mathematical models of temperature fields of a typical bored pile are substantiated and implemented.

3. The proposed mathematical model of a thermodynamic system with a minimum level of error allows replacing a linear heat source into a conventionally cylindrical one, which, in turn, greatly simplifies the implementation of mathematical models in an algorithmic language.

\section{References}

1. A. Mestnikov, A. Egorova, T. Kornilov, A. Kardasevskij, Construction materials, 4 (2009)

2. T. Rad'ko, Investments, construction, real estate as a material basis of modernization and innovative development of the economy (2018)

3. G. Turantaev, Winter concreting of construction structures at non-traditional methods of heat treatment of concrete in thermoactive formwork (2006)

4. R. Ghosh, J. Mustard, Canadian Journal of Civil Engineering, 3 (1983)

5. A. Tran-Le, S. Nguyen, T. Langlet, Energy and Building, 182 (2019)

6. G. Turantaev, Industrial and civil engineering, 4 (2010)

7. M. Ashrabova, Ways to improve the efficiency of irrigated agriculture, 1 (2018)

8. N. Lukuttsova, International journal of applied engineering research, 10 (2015)

9. A. Trinker,Concrete technologies, 2 (2013)

10. I. Pavlova, M. Kocergina, M. Murakaev, Resource and energy efficient technologies in the construction complex of the region, 8 (2017)

11. Z. Bofang, 20: Construction of Mass Concrete in Winter (2014)

12. A. Yudina, R. Oganyan, Architecture and engineering, 2 (2017)

13. M. Berry, J. Johnson, K. McDevitt, Cold Regions Science and Technology, 136 (2017)

14. J. Won, S. Lee, T. Park, K. Nam Construction and Building Materials, 125 (2016)

15. A. Melnik, Procedia Engineering, 206 (2017)

16. Z. Zhou, P. Qiao, Cement and Concrete Composites, 94 (2018)

17. A. Yudina, R. Oganyan, Architecture and Engineering, 2 (2018)

18. D. Anderson, Computational fluid mechanics and heat transfer, 1 (1990)

19. A. Samarskij, Introduction to the theory of difference schemes (1971)

20. N. Yanenko, The method of fractional steps for solving multidimensional problems of mathematical physics (1966) 